\title{
Editorial
}

\section{Gallbladder Cancer Gene Hypermethylation: Genetics or Environment?}

\author{
Henry A. Pitt, MD, and Bruce M. Brenner, MD
}

The article by House et al. ${ }^{1}$ documents the progression of tumor suppressor gene hypermethylation leading to gallbladder cancer. Aberrant methylation was present in $7 \%$ of normal gallbladders, $28 \%$ of gallbladders with chronic cholecystitis secondary to cholelithiasis, and $72 \%$ with cancer. Hypermethylation occurred with equal frequency in cancers from the United States (Johns Hopkins Medical Institutions) and from Chile (Pontificia Universidad Catolica de Chile). The most commonly methylated tumor suppressor genes were p16 (56\%; 50\% United States, 60\% Chile), p73 (28\%; 13\% United States, $40 \%$ Chile, $P<.04)$, and APC (26\%; $42 \%$ United States, $14 \%$ Chile, $P<.03$ ). As with most good studies, this article asks more questions than it answers.

Perhaps the most interesting question raised by this analysis is whether the differences observed between the tumors from the United States and Chile are genetic or environmental. Surprisingly, the manuscript by House et al. ${ }^{1}$ provides no information about the race of the patients. The patients with tumors from the United States and Chile were the same age (median, 60 years) and gender (female, 70\%), and were reasonably well matched by tumor stage. Presumably, most of the patients in the Johns Hopkins group were white, but some may have been black, Asian, or Hispanic. Similarly, the Chilean patients may have been Amerindian, Hispanic, or Maori. A subanalysis comparing gene methylation between whites and Amerindians would have been interesting. The relatively small numbers of patients in this type of subanalysis, however, may have prevented the authors from drawing any meaningful conclusions.

Received July 23, 2003; accepted August 22, 2003.

From the Department of Surgery, Medical College of Wisconsin, Milwaukee, Wisconsin.

Address correspondence to: Henry A. Pitt, MD, Department of Surgery, Medical College of Wisconsin, 9200 West Wisconsin Ave., Milwaukee, WI 53226; Fax: 414-259-9225; E-mail: hapitt@mcw.edu.

Published by Lippincott Williams \& Wilkins @ 2003 The Society of Surgical Oncology, Inc.
The inclusion of patients with normal gallbladders and with chronic cholecystitis secondary to cholelithiasis in the study by House et al. ${ }^{1}$ was important. Although most of these "control" patients, as those with cancer, were female; they were significantly younger, and they were all from Chile. One group that could have been chosen as "normal" controls from the United States were elderly patients with colorectal liver metastases requiring cholecystectomy at the time of liver resection. Similarly, inclusion of elderly patients having cholecystectomy for chronic cholecystitis and cholelithiasis from the United States would have enhanced the analysis.

The statistically significant difference in $A P C$ and $p 73$ gene methylation observed by House et al. ${ }^{1}$ between tumors from the United States and Chile suggests that genetic differences may have led to the unique tumor biology. Recent studies of several common polygenic disorders, however, suggest that approximately one third of these diseases are genetic, whereas two thirds are environmental. ${ }^{2}$ This relative contribution of genetics and environment probably also applies to many cancers. Thus, another interesting question raised by House et al. ${ }^{1}$ is what environmental factors may have differed between their patients from the United States and those from Chile. Environmental candidates include diet, chemicals, radionuclides, and the specifics of gallbladder inflammation.

Multiple dietary factors can play a role in the pathogenesis of biliary as well as other gastrointestinal malignancies. Nitrosamines have been implicated in inhabitants of northeast Thailand with biliary malignancies and in animal models, but probably are not major carcinogens in the United States or Chile. ${ }^{3}$ Capsaicin has been suggested as a potential dietary carcinogen in Chilean gallbladder cancer patients, whereas the high fat Western diet common in the United States may be an important factor in both gallstone formation and in the development of a number of malignancies. Several chemicals, 
including dioxin, also have been suggested to be cofactors in the pathogenesis of biliary malignancies. ${ }^{3}$ Obviously, House et al. ${ }^{1}$ did not have detailed dietary histories of their patients nor information about their exposure to chemicals or radionuclides. Future studies that compare the molecular genetics of Asian, Caucasian, and Amerindian biliary malignancies and document dietary history and environmental exposures would help to answer the basic question regarding the relative role of genetics and environment in gallbladder and bile duct cancers.

Another intriguing question raised by the findings of House et al. ${ }^{1}$ is whether the microenvironment within the gallbladder is the same in patients from the United States and in those from Chile. All patients with cancers from both countries had gallstones, but the number and type of gallstones was not provided. Some data suggest that patients with large solitary gallstones are more susceptible to form cancers. In the United States and at Johns Hopkins, approximately $75 \%$ of patients having cholecystectomy for chronic cholecystitis have cholesterol gallstones. ${ }^{4}$ Similarly, most patients with gallstones in Chile have cholesterol stones and so do Chilean patients with gallbladder cancer (H.A. Pitt, unpublished data, 2002). Clearly, biliary stasis and bacterial infection are microenvironmental factors that predispose to biliary malignancy. Bile salts are known carcinogens, but the composition of bile in patients with gallbladder cancer is unknown. Similarly, the incidence and type of bacteria associated with gallbladder cancer, and the role that bacteria may play in hypermethylation or microsatellite instability remains to be defined.

The interplay of genetic and epigenetic events as well as environmental or ethnic factors certainly has important implications in the pathogenesis of gallbladder and other cancers. As mentioned in the introduction to the paper by House et al. ${ }^{1}$, a number of genetic alterations have been previously described in gallbladder cancer. Comprehensive and focused allelotyping has been done in Chilean patients demonstrating frequent allelic losses on chromosomes 3p, 8p, 9q, and 22q.5 A sequence of events was identified that correlated with progressive histologic changes. Loss of heterozygosity has previously been reported in gallbladder cancer for $p 16^{6}$ and $A P C,{ }^{7}$ loci that are frequently methylated in the present study. In addition, microsatellite instability was found in $17 \%$ of gallbladder cancers in a study from Japan. Inter- estingly, these patients had relatively low levels of allelic loss. ${ }^{7}$ Microsatellite instability was not identified in patients from Italy ${ }^{8}$ or Korea. ${ }^{6}$ In summary, multiple genetic and epigenetic events are implicated in the pathogenesis of gallbladder cancer and seen with varying frequencies in patients from different ethnic backgrounds. The interplay of these changes and the contribution of dietary and other environmental factors in producing these events remain to be elucidated.

In conclusion, the article by House et al. ${ }^{1}$ in this issue of the Annals of Surgical Oncology adds another interesting piece to the cancer puzzle. Their finding that tumor suppressor gene hypermethylation is rare in normal gallbladders, present in $25 \%$ to $30 \%$ of gallbladders with chronic inflammation, and common (70\% to $75 \%)$ in gallbladders with cancer is new and analogous to other cancer progression sequences. Their observation that $p 16$ abnormalities are common in biliary malignancies confirms other reports. Their data suggesting that $A P C$ hypermethylation was more common $(P<.03)$ in tumors from the United States and that $p 73$ hypermethylation was more common $(P<.04)$ in Chilean tumors is unique, however. This finding raises the intriguing possibility that tumor molecular genetics can vary with race; however, a more likely probability is that multiple environmental factors also contribute to unique tumor biology.

\section{REFERENCES}

1. House MG, Wistuba II, Argani P, et al. Progression of gene hypermethylation in gallstone disease leading to gallbladder cancer. Ann Surg Oncol 2003;10:882-9.

2. Nakeeb A, Comouzzie AG, Martin L, et al. Gallstones: genetics verus environment. Ann Surg 2002;235:842-9.

3. Pitt HA, Dooley WC, Yeo CJ, Cameron JL. Malignancies of the biliary tract. Curr Probl Surg 1995;32:1-90.

4. Magnuson TH, Lillemoe KD, Pitt HA. How many Americans will be eligible for biliary lithotripsy? Arch Surg 1989;1254:11951200 .

5. Wistuba II, Maitra A, Carrasco R, et al. High resolution chromosome $3 p, 8 p, 9 q$ and $22 q$ allelotyping analysis in the pathogenesis of gallbladder carcinoma. Br J Cancer 2002;87:432-40.

6. Kim YT, Kim J, Jang YH, et al. Genetic alterations in gallbladder adenoma, dysplasia and carcinoma. Cancer Lett 2001;169:59-68.

7. Yoshida T, Sugai T, Habano W, et al. Microsatellite instability in gallbladder carcinoma: two independent genetic pathways of gallbladder carcinogenesis. J Gastroenterol 2000;35:768-74.

8. Sessa F, Furlan D, Genasetti A, et al. Microsatellite instability and p53 expression in gallbladder carcinomas. Diagn Mol Pathol 2003; 12:96-102. 